\title{
A CASE OF BRAIN GUMMA
}

BY

\author{
M. BIANCHI and C. FRERA
}

\author{
From the Neurosurgical Department of the Istituto Neurologico " C. Besta", Milan, Italy
}

Surgical reports of space-occupying syphilitic granulomas of the brain have always been rare and chemotherapeutic treatment has still further reduced their number. In 1929 Bagdasar pointed out that while from 1908 to 1912 Cushing found five cerebral gummas in 194 neurosurgical operations, from 1913 to 1927 he met with only three of these lesions in 1,500 surgical operations of the brain (Cushing, 1932).

Pre-operative diagnosis of cerebral gummas has always proved extremely difficult for they cause no specific syndromes and the observation that a patient has signs of an expanding intracranial lesion and positive syphilitic reactions provides insufficient grounds for diagnosis. Even the therapeutic criterion is untenable, since, while chemotherapy is effective in preventing the formation of gummas, it may be useless in their treatment. These difficulties in diagnosis still exist to-day, and, as in a case of cerebral gumma observed by us, peculiar results were obtained by carotid angiography, which has not, as far as we know, been applied to this disease before, it seems to us that the case is worth reporting for its possible diagnostic interest.

\section{Case Report}

A man of 35 (P. R. No. 19573) was admitted to the Milan Neurological Institute in February, 1954. The patient remembered no other illness except a pharyngeal diphtheria at the age of 8 ; he was a heavy drinker. Twenty months before being taken into the Institute he had suffered a slight injury which produced only a " stunned" feeling, lasting no more than a few moments. Two months after this injury the patient began suffering from attacks of left-sided temporal hemicrania lasting two or three days and separated by free intervals of eight to 10 days. About nine months later he had a generalized epileptic seizure, which recurred twice during the next two months. There followed a period of acute mental confusion, which was put down to excess of alcohol and the patient was sent to a hospital where he was treated for chronic alcoholism for a month. After this episode the patient's character changed greatly: first he became very euphoric, then came periods of apathy alternating with others in which he was irascible and impatient, and others again in which he was once more euphoric and highly active in a disorderly manner. A month before he came to the Institute, his mental disturbance became more serious, the headaches more intense, spreading to the right side; there was somnolence and frequent yawning.

On examination the patient was apathetic and confused; his memory for recent events was obviously deficient and his power of concentration quickly exhausted; there were no speech disturbances. The outstanding results of neurological examination were painful cranial percussion on the right side and bilateral anosmia. The left pupil was wider than the right; both reacted sluggishly to light but normally to accommodation and convergence. Visual acuity was $10 / 10$ in both eyes; he had a left inferior homonymous quadrantanopsia and bilateral papilloedema of 0.5 diopters on the right side and 2 diopters on the left. External ocular movements were normal. He had a left facial hypokinesia, but no other cranial nerve disturbances. He had slight hypotonia of the limbs of the right side without signs of motor or sensory impairment. The deep reflexes were brisker on the right side, the Babinsky sign was more evident on the right side where there was also a positive Gordon reflex, and a bilateral grasping reflex. No relevant symptoms were evident on routine clinical examination of the heart, lungs, and abdomen.

Blood pressure was $120 / 80 \mathrm{~mm}$. Hg. Urine was normal. Wassermann's and Kahn's syphilitic reactions were markedly positive.

An E.E.G. revealed slow, wide waves more marked on the right fronto-temporal region but spreading to the anterior half of the skull on both sides.

Radiographs of the skull disclosed a sellar decalcification and a posterior pineal shift. Ventriculography contributed nothing owing to poor filling of the ventricular system. Carotid angiography was performed on both sides: in the antero-posterior view the anterior cerebral arteries were displaced to the right, but the most interesting finding was the appearance of these vessels in the lateral view. They were very slender and irregularly injected by the contrast medium; their branches also were of identical appearance and were only faintly visible.

A diagnosis of cerebral gumma was tentatively advanced on the results of the serological examination and the arteriographic findings, but owing to the papilloedema it was thought wiser not to await the possible beneficial effects of chemotherapy. 


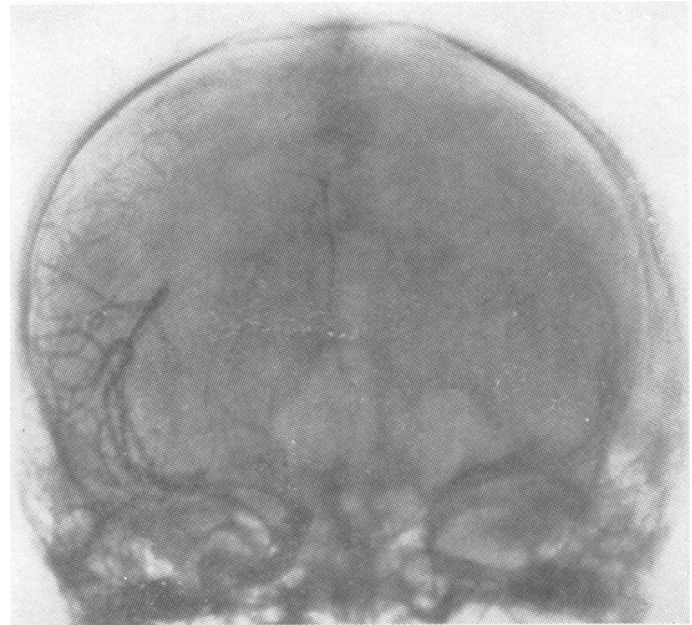

FIG. 1

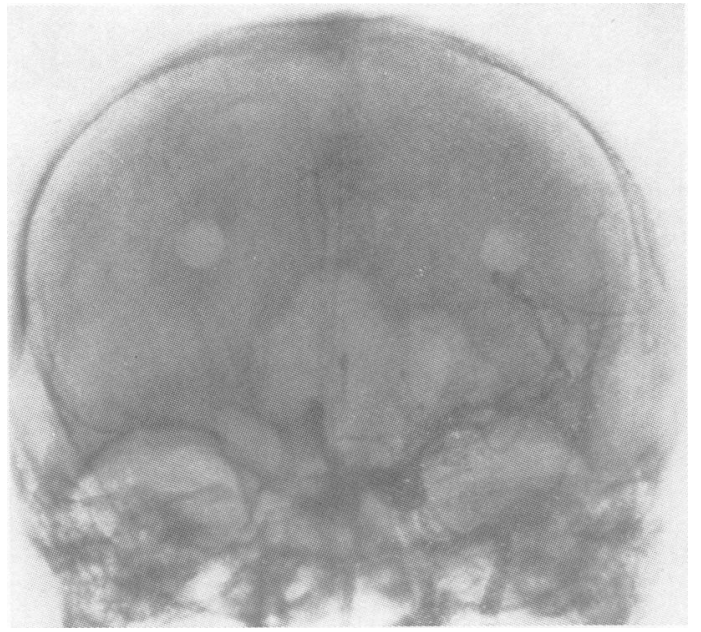

FIG. 2

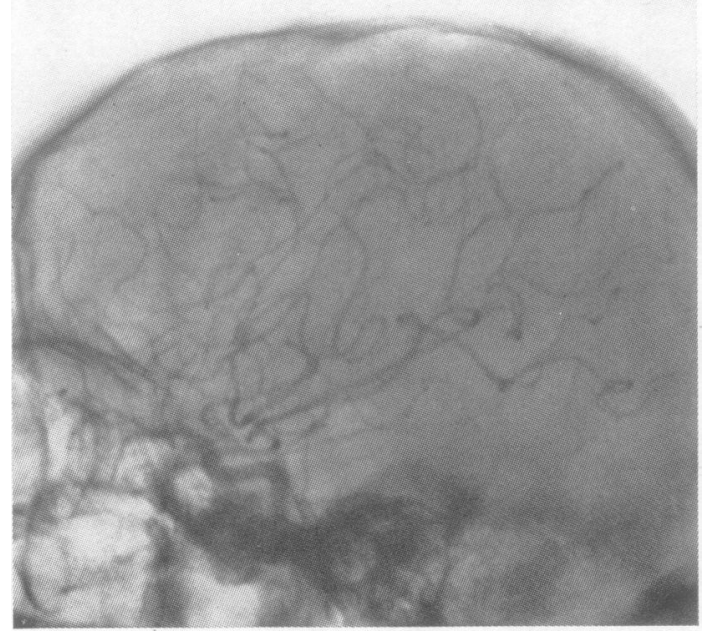

Fig. 3
Operation.-On March 9, 1954, under local (xilocain) anaesthesia both frontal lobes were exposed with a frontal bilateral flap hinged to the left side. Their upper aspect appeared normal but on exploring in the interhemispheric fissure the left frontal lobe was found to be adherent to the falx and the floor of the anterior fossa: the left anterior cerebral artery was whitish. The cerebral substance was cut at the edges of the meningocerebral adhesion and a hard mass of irregular form and of the size of a hen's egg was found in the left frontal lobe. Thanks to its rather sharp outline it could be completely removed with very little loss of blood. During the removal the left anterior cerebral artery, running at the upper edge of the mass, was cut and appeared to be incompletely thrombosed as blood flowed slowly and did not spurt from it. After closing the dura mater, the bone flap was replaced and the galea and skin sutured with silk.

Microscopical Examination.-The section of the mass removed, stained with haematoxylin and eosin, showed a central core of coagulation necrosis and a peripheral area of granulomatous tissue. This was composed of a fine network of collagen fibrils and of small blood vessels which were sometimes ectasic and with sinusoid features. A dense infiltrate filled this network; it chiefly consisted of lymphocytes and plasma cells, but there were also many big mononucleated cells of the macro phagic type and foam cells were grouped here and there into xanthomatous areas. In some places the demarcation between the necrotic core and the granulomatous areas was sharp, but in other places they merged into one another through a transitional area in which fibrils ano cells showed a definite swelling. In this area there were neither epithelioid nor giant cells. In Levaditi's ants. Wright's silver stain many spirillar images appearede. against an unstained background.

Progress.-After an uneventful post-operative period chemotherapeutic treatment (penicillin and mercuriccyanide) was instituted; the papilloedema gradually subsided and the patient was discharged without relevant neurological findings on May 20, 1955. At successive out-patient examinations, he was always found psychically alert and no further epileptic fits were reported; the Wassermann and Kahn reactions remained strongly positive.

The positive serological syphilitic reactions and the macroscopical and microscopical aspects of the lesion, similar to those reported by Gross, Stein, and Myerson (1942), made the diagnosis of cerebral gumma quite acceptable.

The most interesting point in our case is the result of carotid angiography. This examination showed a localized expanding lesion and alterations indicating

FIGS. 1 and 2.-Right and left carotid angiograms, antero-posterior view. The anterior cerebral arteries are slightly displaced to the right side.

FIG. 3.-Left carotid angiogram, lateral view. The anterior cerebral arteries are slender and irregularly filled. 


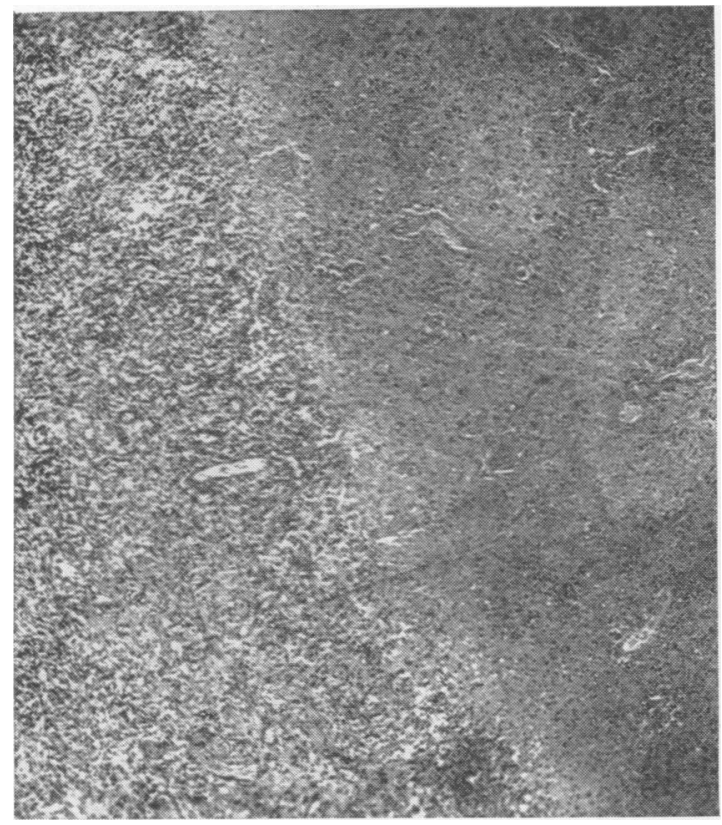

FIG. 4.-Central area of necrosis surrounded by a peripheral zone of granulomatous tissue. Haematoxylin and eosin $\times 72$.

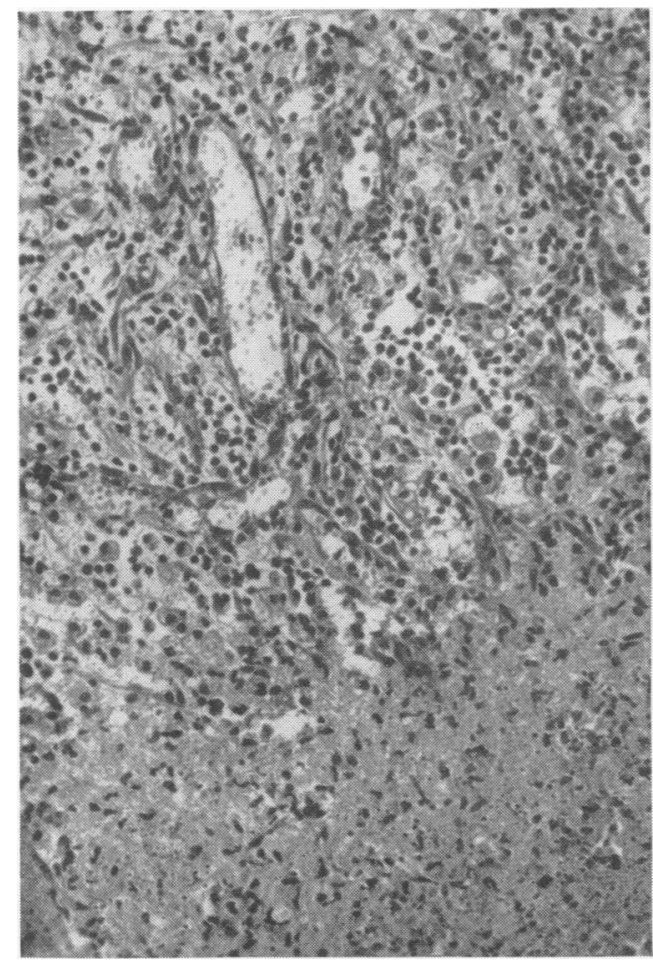

Fig. 5.-Granulomatous tissue. Haematoxylin and eosin. $\times 195$.

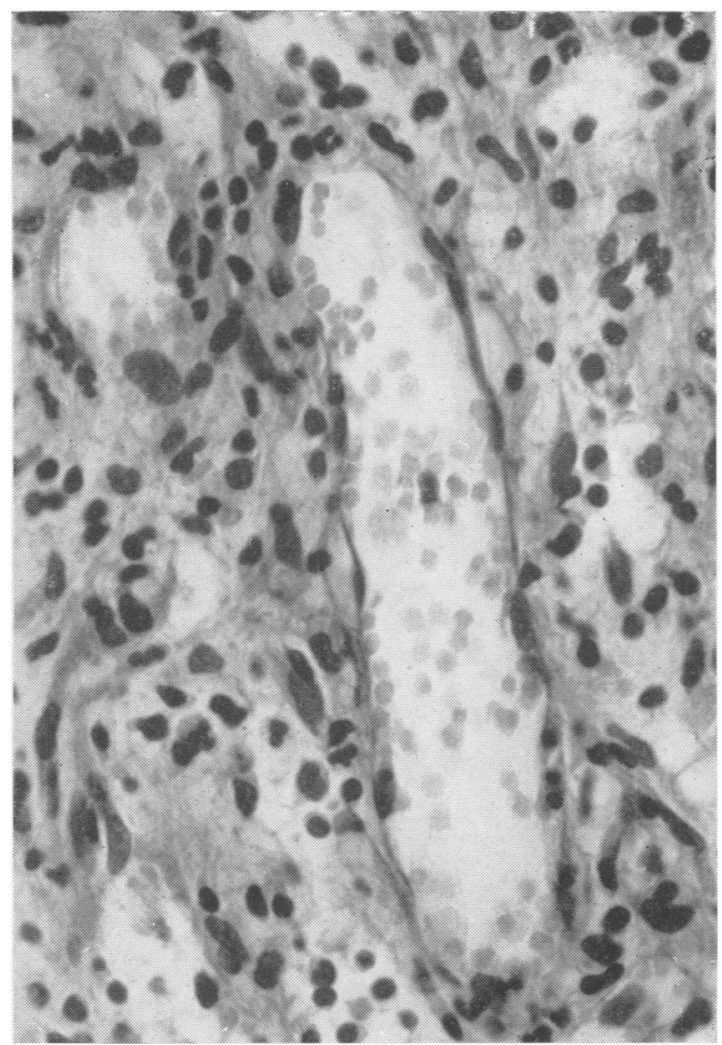

FIG. 6.-The granulomatous tissue at a higher magnification having a network of collagen fibrils and small blood vessels infiltrated with lymphocytes and plasma cells. Haematoxylin and eosin. $\times 540$.

arteritis in the neighbouring vessels. If similar results are found in other cases of gumma, this particular angiographic finding may be considered characteristic of syphilitic granulomas of the brain. From the point of view of treatment the known insensitivity of cerebral gumma to chemotherapy calls for their surgical removal without delay, and in our case the existence of a bilateral papilloedema provided another reason for urgent intervention.

\section{Summary}

A case of surgically treated cerebral gumma is described. The most interesting point in this case is the result of carotid angiography which showed a space-occupying lesion with arteritic alterations in the neighbouring vessels.

\section{REFERENCES}

Bagdasar, D. (1929) . Rev, neurol (Paris), $2,1$.

Cushing, H. (1932). Intracranial Tumours. Thomas, Springfield, Gross, S. W., Stein, A., and Myerson, P. G. (1942). Amer. J. Surg., $58,78$. 